\title{
Epistemic Analysis of Strategic Games with Arbitrary Strategy Sets
}

\author{
Krzysztof R. Apt \\ CWI, Amsterdam, the Netherlands \\ and University of Amsterdam
}

\begin{abstract}
We provide here an epistemic analysis of arbitrary strategic games based on the possibility correspondences. Such an analysis calls for the use of transfinite iterations of the corresponding operators. Our approach is based on Tarski's Fixpoint Theorem and applies both to the notions of rationalizability and the iterated elimination of strictly dominated strategies.
\end{abstract}

\section{Introduction}

Epistemic analysis of strategic games (in short, games) aims at predicting the choices of rational players in the presence of (partial or common) knowledge or belief of the behaviour of other players. Most often it focusses on the iterated elimination of never best responses (a notion termed as rationalizability), and the iterated elimination of strictly dominated strategies (IESDS).

Starting with Aumann [1987], Brandenburger and Dekel [1987] and Tan and Werlang [1988] a large body of literature arose that investigates the epistemic foundations of rationalizability by modelling the reasoning employed by players in choosing their strategies. Such an analysis, based either on possibility correspondences and partition spaces, or Harsanyi type spaces, is limited either to finite or compact games with continuous payoffs, or to two-player games, see, e.g., Battigalli and Bonanno [1999] or Ely and Peski [2006].

In turn, in the case of IESDS the epistemic analysis has focussed on finite games (with an infinite hierarchy of beliefs) and strict dominance either by pure or by mixed strategies, see, e.g. Brandenburger, Friedenberg and Keisler [2004].

In this paper we provide an epistemic analysis of arbitrary strategic games based on the possibility correspondences. More specifically, denote by $\operatorname{RAT}(\bar{\phi})$ the property that each player $i$ uses a monotonic property $\phi_{i}$ to select his strategy ('each player $i$ is $\phi_{i}$-rational'). Then the following sets of strategy profiles coincide:

- those that the players choose in the states in which $\operatorname{RAT}(\bar{\phi})$ is common knowledge,

- those that the players choose in the states in which $\operatorname{RAT}(\bar{\phi})$ is true and is common belief,

- those that remain after the iterated elimination of the strategies that are not $\phi_{i}$-optimal.

This requires that transfinite iterations of the strategy elimination are allowed and covers the usual notion of rationalizability and a 'global' version ${ }^{1}$ of the iterated elimination of strictly dominated strategies. For the customary, 'local' version of the iterated elimination of strictly dominated strategies (that is defined using a non-monotonic property) we justify the statement

common knowledge of rationality implies that the players will choose only strategies that survive the iterated elimination of strictly dominated strategies

for arbitrary games and transfinite iterations of the elimination process. Rationality refers here to the concept studied in Bernheim [1984].

Our results complement the findings of Lipman [1991] in which transfinite ordinals are used in a study of limited rationality and Lipman [1994], where a two-player game is constructed for which the $\omega_{0}$ (the first infinite ordinal) and $\omega_{0}+1$ iterations of the rationalizability operator of Bernheim [1984] differ. In turn, Heifetz and Samet [1998] show that in general arbitrary ordinals are necessary in the epistemic analysis of strategic games based on the partition spaces. Further, as

\footnotetext{
${ }^{1}$ The concepts of 'global' and 'local' versions are clarified in Section 3.
} 
argued in Chen, Long and Luo [2005], the notion of IESDS à la Milgrom and Roberts [1990], when used for arbitrary games, also requires transfinite iterations of the underlying operator.

The relevance of monotonicity in the context of epistemic analysis of finite strategic games has already been pointed out in van Benthem [2007], where the notions of strict dominance and rationalizability are studied using a public announcement logic.

\section{Preliminaries}

In this section we recall basic results concerning operators on a complete lattice and the relevant notions concerning strategic games.

\section{$2.1 \quad$ Operators}

Consider a fixed complete lattice $(D, \subseteq)$ with the largest element $T$. In what follows we use ordinals and denote them by $\alpha, \beta, \gamma$. Given a, possibly transfinite, sequence $\left(G_{\alpha}\right)_{\alpha<\gamma}$ of elements of $D$ we denote their join and meet respectively by $\bigcup_{\alpha<\gamma} G_{\alpha}$ and $\bigcap_{\alpha<\gamma} G_{\alpha}$.

Definition 1 Let $T$ be an operator on $(D, \subseteq)$, i.e., $T: D \rightarrow D$.

- We call $T$ monotonic if for all $G_{1}, G_{2}$

$$
G_{1} \subseteq G_{2} \text { implies } T\left(G_{1}\right) \subseteq T\left(G_{2}\right)
$$

- We call $T$ contracting if for all $G$

$$
T(G) \subseteq G
$$

- We say that an element $G$ is a fixpoint of $T$ if $G=T(G)$ and a post-fixpoint of $T$ if $G \subseteq T(G)$.

- We define by transfinite induction a sequence of elements $T^{\alpha}$ of $D$, where $\alpha$ is an ordinal, as follows:

$$
\begin{aligned}
& -T^{0}:=\top \\
& -T^{\alpha+1}:=T\left(T^{\alpha}\right), \\
& \text { - for all limit ordinals } \beta, T^{\beta}:=\bigcap_{\alpha<\beta} T^{\alpha} .
\end{aligned}
$$

- We call the least $\alpha$ such that $T^{\alpha+1}=T^{\alpha}$ the closure ordinal of $T$ and denote it by $\alpha_{T}$. We call then $T^{\alpha_{T}}$ the outcome of (iterating) $T$ and write it alternatively as $T^{\infty}$.

So an outcome is a fixpoint reached by a transfinite iteration that starts with the largest element. In general, the outcome of an operator does not need to exist but we have the following classic result due to Tarski $[1955] .^{2}$

Tarski's Fixpoint Theorem Every monotonic operator $T$ on $(D, \subseteq)$ has an outcome, i.e., $T^{\infty}$ is welldefined. Moreover,

$$
T^{\infty}=\nu T=\cup\{G \mid G \subseteq T(G)\},
$$

where $\nu T$ is the largest fixpoint of $T$.

In contrast, a contracting operator does not need to have a largest fixpoint. But we have the following obvious observation.

Note 1 Every contracting operator $T$ on $(D, \subseteq)$ has an outcome, i.e., $T^{\infty}$ is well-defined.

In Section 5 we shall need the following lemma.

Lemma 1 Consider two operators $T_{1}$ and $T_{2}$ on $(D, \subseteq)$ such that

- for all $G, T_{1}(G) \subseteq T_{2}(G)$,

- $T_{1}$ is monotonic,

- $T_{2}$ is contracting.

Then $T_{1}^{\infty} \subseteq T_{2}^{\infty}$.

Proof. We first prove by transfinite induction that for all $\alpha$

$$
T_{1}^{\alpha} \subseteq T_{2}^{\alpha}
$$

By the definition of the iterations we only need to consider the induction step for a successor ordinal. So suppose the claim holds for some $\alpha$. Then by the first two assumptions and the induction hypothesis we have the following string of inclusions and equalities:

$$
T_{1}^{\alpha+1}=T_{1}\left(T_{1}^{\alpha}\right) \subseteq T_{1}\left(T_{2}^{\alpha}\right) \subseteq T_{2}\left(T_{2}^{\alpha}\right)=T_{2}^{\alpha+1} .
$$

This shows that for all $\alpha$ (1) holds. By Tarski's Fixpoint Theorem and Note 1 the outcomes of $T_{1}$ and $T_{2}$ exist, which implies the claim.

\subsection{Strategic games}

Given $n$ players $(n>1)$ by a strategic game (in short, a game) we mean a sequence $\left(S_{1}, \ldots, S_{n}, p_{1}, \ldots, p_{n}\right)$, where for each $i \in[1 . . n]$

- $S_{i}$ is the non-empty set of strategies (sometimes called actions) available to player $i$,

\footnotetext{
${ }^{2}$ We use here its 'dual' version in which the iterations start at the largest and not at the least element of a complete lattice.
} 
- $p_{i}$ is the payoff function for the player $i$, so $p_{i}: S_{1} \times \ldots \times S_{n} \rightarrow \mathcal{R}$, where $\mathcal{R}$ is the set of real numbers.

We denote the strategies of player $i$ by $s_{i}$, possibly with some superscripts. Given $s \in S_{1} \times \ldots \times S_{n}$ we denote the $i$ th element of $s$ by $s_{i}$, write sometimes $s$ as $\left(s_{i}, s_{-i}\right)$, and use the following standard notation:

- $s_{-i}:=\left(s_{1}, \ldots, s_{i-1}, s_{i+1}, \ldots, s_{n}\right)$,

- $S_{-i}:=S_{1} \times \ldots \times S_{i-1} \times S_{i+1} \times \ldots \times S_{n}$.

Given a finite non-empty set $A$ we denote by $\Delta A$ the set of probability distributions over $A$ and call any element of $\Delta S_{i}$ a mixed strategy of player $i$.

In what follows we assume an initial strategic game

$$
H:=\left(T_{1}, \ldots, T_{n}, p_{1}, \ldots, p_{n}\right) .
$$

A restriction of $H$ is a sequence $\left(S_{1}, \ldots, S_{n}\right)$ such that $S_{i} \subseteq T_{i}$ for $i \in[1 . . n]$. We identify the restriction $\left(T_{1}, \ldots, T_{n}\right)$ with $H$. We shall focus on the complete lattice that consists of the set of all restrictions of the game $H$ ordered by the componentwise set inclusion:

$$
\left(S_{1}, \ldots, S_{n}\right) \subseteq\left(S_{1}^{\prime}, \ldots, S_{n}^{\prime}\right) \text { iff } S_{i} \subseteq S_{i}^{\prime} \text { for all } i \in[1 . . n] .
$$

So $H$ is the largest element in this lattice and $\bigcup_{\alpha<\gamma}$ and $\bigcap_{\alpha<\gamma}$ are the customary set-theoretic operations on the restrictions.

Consider now a restriction $G:=\left(S_{1}, \ldots, S_{n}\right)$ of $H$ and two strategies $s_{i}, s_{i}^{\prime}$ from $T_{i}$ (so not necessarily from $S_{i}$ ). We say that $s_{i}$ is strictly dominated on $G$ by $s_{i}^{\prime}$ if

$$
\forall s_{-i} \in S_{-i} p_{i}\left(s_{i}^{\prime}, s_{-i}\right)>p_{i}\left(s_{i}, s_{-i}\right),
$$

and write then $s_{i}^{\prime} \succ_{G} s_{i}$.

In the case of finite games, once the payoff function is extended in the expected way to mixed strategies, the relation $\succ_{G}$ between a mixed strategy and a pure strategy is defined in the same way.

Further, given a restriction $G^{\prime}:=\left(S_{1}^{\prime}, \ldots, S_{n}^{\prime}\right)$ of $H$, we say that the strategy $s_{i}$ from $T_{i}$ is a best response in $G^{\prime}$ to some belief $\mu_{i}$ held in $G$ if

$$
\forall s_{i}^{\prime} \in S_{i}^{\prime} p_{i}\left(s_{i}, \mu_{i}\right) \geq p_{i}\left(s_{i}^{\prime}, \mu_{i}\right) .
$$

A belief held in $G:=\left(S_{1}, \ldots, S_{n}\right)$ can be

- a joint strategy of the opponents of player $i$ in $G$ (i.e., $s_{-i} \in S_{-i}$ ),

- or, in the case the game is finite, a joint mixed strategy of the opponents of player $i$ (i.e., $\left(m_{1}, \ldots, m_{i-1}, m_{i+1}, \ldots, m_{n}\right)$, where $m_{j} \in \Delta S_{j}$ for all $j$ ),
- or a correlated strategy of the opponents of player $i$ (i.e., $m \in \Delta S_{-i}$ ).

Every joint mixed strategy of the opponents of player $i$ can be identified with their correlated strategy.

\section{$3 \quad$ Set up}

The assumption that each player is rational is one of the basic stipulations within the framework of strategic games. However, rationality can be differently interpreted by different players. ${ }^{3}$ This may for example mean that a player

- does not choose a strategy strictly dominated by another pure/mixed strategy,

- chooses only best replies to the (beliefs about the) strategies of the opponents.

In this paper we are interested in analyzing situations in which each player pursues his own notion of rationality, more specifically the situations in which this information is common knowledge or common belief. As a special case we cover then the usually analyzed situation in which all players use the same notion of rationality.

Given player $i$ in a strategic game $H$ := $\left(T_{1}, \ldots, T_{n}, p_{1}, \ldots, p_{n}\right)$ we formalize his notion of rationality as a property $\phi_{i}\left(s_{i}, G, G^{\prime}\right)$ that holds between a state $s_{i} \in T_{i}$ and restrictions $G$ and $G^{\prime}$ of $H$. Intuitively, $\phi\left(s_{i}, G, G^{\prime}\right)$ holds if $s_{i}$ is an 'optimal' strategy for player $i$ within the restriction $G$ in the context of $G^{\prime}$, assuming that he uses the property $\phi$ to select optimal strategies.

Here are some examples of the property $\phi$ which show that the abovementioned rationality notions can be formalized in a number of natural ways:

- $s d\left(s_{i}, G, G^{\prime}\right)$ that holds iff the strategy $s_{i}$ of player $i$ is not strictly dominated on $G$ by any strategy from the restriction $G^{\prime}:=\left(S_{1}^{\prime}, \ldots, S_{n}^{\prime}\right)$ of $H$ (i.e., $\left.\neg \exists s_{i}^{\prime} \in S_{i}^{\prime} s_{i}^{\prime} \succ_{G} s_{i}\right)$,

- (assuming $H$ is finite) $\operatorname{msd}\left(s_{i}, G, G^{\prime}\right)$ that holds iff the strategy $s_{i}$ of player $i$ is not strictly dominated on $G$ by any of its mixed strategy from the restriction $G^{\prime}:=\left(S_{1}^{\prime}, \ldots, S_{n}^{\prime}\right)$ of $H$, (i.e., $\left.\neg \exists m_{i}^{\prime} \in \Delta S_{i}^{\prime} m_{i}^{\prime} \succ_{G} s_{i}\right)$,

\footnotetext{
${ }^{3}$ This matter is obfuscated by the fact that the etymologically related noun 'rationalizability' stands by now for the concept introduced in Bernheim [1984] and Pearce [1984] that refers to the outcome of iterated elimination of never best responses.
} 
- $\operatorname{br}\left(s_{i}, G, G^{\prime}\right)$ that holds iff the strategy $s_{i}$ of player $i$ is a best response in the restriction $G^{\prime}:=$ $\left(S_{1}^{\prime}, \ldots, S_{n}^{\prime}\right)$ of $H$ to some belief $\mu_{i}$ held in $G$ (i.e., for some belief $\mu_{i}$ held in $G, \forall s_{i}^{\prime} \in S_{i}^{\prime} p_{i}\left(s_{i}, \mu_{i}\right) \geq$ $\left.p_{i}\left(s_{i}^{\prime}, \mu_{i}\right)\right)$.

Two natural possibilities for $G^{\prime}$ are $G^{\prime}=H$ or $G^{\prime}=$ $G$. We then abbreviate $\phi\left(s_{i}, G, H\right)$ to $\phi^{g}\left(s_{i}, G\right)$ and $\phi\left(s_{i}, G, G\right)$ to $\phi^{l}\left(s_{i}, G\right)$ and henceforth focus on the binary properties $\phi(\cdot, \cdot)$. (The superscript ' $g$ ' stands for 'global' and ' $l$ ' for 'local'.)

We say that the property $\phi(\cdot, \cdot)$ (used by player $i$ ) is monotonic if for all restrictions $G$ and $G^{\prime}$ of $H$ and $s_{i} \in T_{i}$

$$
G \subseteq G^{\prime} \text { and } \phi\left(s_{i}, G\right) \text { implies } \phi\left(s_{i}, G^{\prime}\right) .
$$

Each sequence of properties $\bar{\phi}:=\left(\phi_{1}, \ldots, \phi_{n}\right)$ determines an operator $T_{\bar{\phi}}$ on the restrictions of $H$ defined by

$$
T_{\bar{\phi}}(G):=\left(S_{1}^{\prime}, \ldots, S_{n}^{\prime}\right),
$$

where $G:=\left(S_{1}, \ldots, S_{n}\right)$ and for all $i \in[1 . . n]$

$$
S_{i}^{\prime}:=\left\{s_{i} \in S_{i} \mid \phi_{i}\left(s_{i}, G\right)\right\} .
$$

Since $T_{\bar{\phi}}$ is contracting, by Note 1 it has an outcome, i.e., $T_{\bar{\phi}}^{\infty}$ is well-defined. Moreover, if each $\phi_{i}$ is monotonic, then $T_{\bar{\phi}}$ is monotonic and by Tarski's Fixpoint Theorem its largest fixpoint $\nu T_{\bar{\phi}}$ exists and equals $T_{\bar{\phi}}^{\infty}$.

Intuitively, $T_{\bar{\phi}}(G)$ is the result of removing from $G$ all strategies that are not $\phi_{i}$-optimal. So the outcome of $T_{\bar{\phi}}$ is the result of the iterated elimination of strategies that for player $i$ are not $\phi_{i}$-optimal, where $i \in[1 . . n]$.

When each property $\phi_{i}$ equals $\phi$, we write $T_{\phi}$ instead of $T_{\bar{\phi}}$. The natural examples of such an iterated elimination of strategies that were discussed in the literature are: ${ }^{4}$

- iterated elimination of strategies that are strictly dominated by another strategy;

This corresponds to the iterations of the $T_{s d^{l}}$ operator in the case of Dufwenberg and Stegeman [2002]) and of the $T_{s d^{g}}$ operator in the case of Chen, Long and Luo [2005].

- (for finite games) iterated elimination of strategies that are strictly dominated by a mixed strategy;

This is the customary situation studied starting with Luce and Raiffa [1957] that corresponds to the iterations of the $T_{m s d^{l}}$ operator.

\footnotetext{
${ }^{4}$ The reader puzzled by the existence of multiple definitions for the apparently uniquely defined concepts is encouraged to consult Apt [2007].
}

- iterated elimination of strategies that are never best responses to some belief;

This corresponds to the iterations of the $T_{b r} g$ operator in the case of Bernheim [1984] and the $T_{b r} l$ operator in the case of Pearce [1984], in each case for an appropriate set of beliefs.

Usually only the first $\omega_{0}$ iterations of the corresponding operator $T$ are considered, i.e., one studies $T^{\omega_{0}}$, that is $\bigcap_{i<\omega_{0}} T^{i}$, and not $T^{\infty}$.

In the next section we assume that each player $i$ employs some property $\phi_{i}$ to select his strategies and analyze the situation in which this information is common knowledge. To determine which strategies are then selected by the players we shall use the $T_{\bar{\phi}}$ operator. We shall also explain why in general transfinite iterations are necessary.

\section{Two theorems}

To proceed further we need to recall some basic facts concerning the epistemic analysis of strategic games. The approach taken below is based on the partition spaces, or more generally possibility correspondences. We follow here the exposition of Battigalli and Bonanno [1999].

Given the initial game $H=\left(T_{1}, \ldots, T_{n}, p_{1}, \ldots, p_{n}\right)$ we assume a space $\Omega$ of states such that in each state $\omega \in \Omega$ each player $i$ chooses the strategy $s_{i}(\omega) \in T_{i}$. We assume that for $i \in[1 . . n]$ we have $|\Omega| \geq\left|T_{i}\right|$, where for a set $A$ we denote its cardinality by $|A|$. A natural example of $\Omega$ satisfying this assumption is the set of joint strategies in the game $H$. Then given a state $\omega:=s$ we simply have $s_{i}(\omega)=s_{i}$.

A possibility correspondence is a mapping from $\Omega$ to $\mathcal{P}(\Omega)$. We consider three properties of a possibility correspondence $P$ :

(i) for all $\omega, P(\omega) \neq \emptyset$,

(ii) for all $\omega$ and $\omega^{\prime}, \omega^{\prime} \in P(\omega)$ implies $P\left(\omega^{\prime}\right)=P(\omega)$,

(iii) for all $\omega, \omega \in P(\omega)$.

If the possibility correspondence satisfies properties (i) and (ii), we call it a belief correspondence and if it satisfies properties (i)-(iii), we call it a knowledge correspondence. ${ }^{5}$

In the latter case the correspondence $P$ yields a partition $\{P(\omega) \mid \omega \in \Omega\}$ of $\Omega$.

\footnotetext{
${ }^{5}$ In the modal logic terminology a belief correspondence is a frame for the modal logic KD45 and a knowledge correspondence is a frame for the modal logic S5, see, e.g. Blackburn, de Rijke and Venema [2001].
} 
We assume that each player $i$ has a possibility correspondence $P_{i}$ on $\Omega$. Recall that an event is a subset of $\Omega$, and that an event $F$ is evident if for all $\omega \in F$ we have $P_{i}(\omega) \subseteq F$ for all $i \in[1 . . n]$.

Following Aumann [1976] if each $P_{i}$ is a knowledge correspondence, we say that an event $E$ is a common knowledge in the state $\omega \in \Omega$ if for some evident event $F$ we have $\omega \in F \subseteq E$. We write then $\omega \in K^{*} E$.

Finally, (using a characterization of Monderer and Samet [1989]) if each $P_{i}$ is a belief correspondence, we say that an event $E$ is a common belief in the state $\omega \in \Omega$ if for some evident event $F$ we have $\omega \in$ $F \subseteq B E$, where $B E=\left\{\omega \in \Omega \mid \forall i \in[1 . . n] P_{i}(\omega) \subseteq E\right\}$. We write then $\omega \in B^{*} E$.

Each event $E$ determines a restriction $G_{E}$ of $H$ defined by $G_{E}:=\left(S_{1}, \ldots, S_{n}\right)$, where for all $j \in[1 . . n]$

$$
S_{j}:=\left\{s_{j}\left(\omega^{\prime}\right) \mid \omega^{\prime} \in E\right\} .
$$

In particular, when player $i$ knows (respectively, believes) that the state is in $P_{i}(\omega)$, the restriction $G_{P_{i}(\omega)}$ represents his knowledge (respectively, his belief) about the players' strategies.

Given now a property $\phi_{i}(\cdot, G)$ that player $i$ uses to select his strategies in the restriction $G$ of $H$, we say that player $i$ is $\phi_{i}$-rational in the state $\omega$ if $\phi_{i}\left(s_{i}(\omega), G_{P_{i}(\omega)}\right)$ holds. Intuitively, if the state of the world is $\omega$, player $i$ only knows (respectively, believes) that the state of the world is in $P_{i}(\omega)$. So $G_{P_{i}(\omega)}$ is the game he knows (respectively, believes in). Hence $\phi_{i}\left(s_{i}(\omega), G_{P_{i}(\omega)}\right)$ captures the idea that if player $i$ uses $\phi_{i}(\cdot, \cdot)$ to select his optimal strategy in the game he is 'aware of', then in the state $\omega$ he indeed acts 'rationally'.

We are interested in the strategies selected by each player in the states in which it is common knowledge (or true and common belief) that each player $i$ is $\phi_{i^{-}}$ rational. To this end we introduce the following set of states $^{6}$ :

$\boldsymbol{\operatorname { R A T }}(\bar{\phi}):=\left\{\omega \in \Omega \mid\right.$ each player $i$ is $\phi_{i}$-rational in $\left.\omega\right\}$

and focus on the following two sets of states:

$$
\begin{aligned}
C K(\bar{\phi}):=\{\omega \in \Omega \mid & \text { for some knowledge } \\
& \text { correspondences } P_{1}, \ldots, P_{n} \\
& \left.\omega \in K^{*} \mathbf{R A T}(\bar{\phi})\right\} \\
C B(\bar{\phi}):=\{\omega \in \Omega \mid & \text { for some belief } \\
& \text { correspondences } P_{1}, \ldots, P_{n} \\
& \left.\omega \in \mathbf{R A T}(\bar{\phi}) \text { and } \omega \in B^{*} \mathbf{R A T}(\bar{\phi})\right\}
\end{aligned}
$$

\footnotetext{
${ }^{6} \boldsymbol{R} \mathbf{A T}(\bar{\phi})$ is always used in the context of specific possibility correspondences.
}

and the corresponding restrictions $G_{C K(\bar{\phi})}$ and $G_{C B(\bar{\phi})}$ of $H$.

The following result then characterizes for arbitrary strategic games the restrictions $G_{C K(\bar{\phi})}$ and $G_{C B(\bar{\phi})}$ in terms of the operator $T_{\bar{\phi}}$.

Theorem 1 Suppose that each property $\phi_{i}$ is monotonic. Then

$$
G_{C K(\bar{\phi})}=G_{C B(\bar{\phi})}=T_{\bar{\phi}}^{\infty} .
$$

Proof. We prove three inclusions.

(i) $G_{C K(\bar{\phi})} \subseteq G_{C B(\bar{\phi})}$.

This inclusion (for an arbitrary $\bar{\phi}$ ) is an immediate consequence of the following alternative characterization of common knowledge due to Monderer and Samet [1989]: if each $P_{i}$ is a knowledge correspondence, an event $E$ is a common knowledge in the state $\omega \in \Omega$ if for some evident event $F$ we have $\omega \in F \subseteq K E$, where $K E=\left\{\omega \in \Omega \mid \forall i \in[1 . . n] P_{i}(\omega) \subseteq E\right\}$.

(ii) $G_{C B(\bar{\phi})} \subseteq T_{\bar{\phi}}^{\infty}$.

Take a strategy $s_{i}$ that is an element of the $i$ th component of $G_{C B(\bar{\phi})}$. So $s_{i}=s_{i}(\omega)$ for some $\omega \in C B(\bar{\phi})$. Then $\omega \in \boldsymbol{R A T}(\bar{\phi})$ and $\omega \in B^{*} \boldsymbol{R A T}(\bar{\phi})$. The latter implies that for some evident event $F$

$$
\omega \in F \subseteq\left\{\omega^{\prime} \in \Omega \mid \forall i \in[1 . . n] P_{i}\left(\omega^{\prime}\right) \subseteq \operatorname{RAT}(\bar{\phi})\right\} .
$$

Take now an arbitrary $\omega^{\prime} \in F \cap \mathbf{R A T}(\bar{\phi})$ and $i \in[1 . . n]$. Since $\omega^{\prime} \in \boldsymbol{R A T}(\bar{\phi})$, player $i$ is $\phi_{i}$-rational in $\omega^{\prime}$, i.e., $\phi_{i}\left(s_{i}\left(\omega^{\prime}\right), G_{P_{i}\left(\omega^{\prime}\right)}\right)$ holds. But $F$ is evident, so $P_{i}\left(\omega^{\prime}\right) \subseteq F$. Moreover by $(2) \quad P_{i}\left(\omega^{\prime}\right) \subseteq \mathbf{R A T}(\bar{\phi})$, so $P_{i}\left(\omega^{\prime}\right) \subseteq F \cap \mathbf{R A T}(\bar{\phi})$. Hence $G_{P_{i}\left(\omega^{\prime}\right)} \subseteq G_{F \cap \mathbf{R A T}(\bar{\phi})}$ and by the monotonicity of $\phi_{i}$ we conclude that $\phi_{i}\left(s_{i}\left(\omega^{\prime}\right), G_{F \cap \mathbf{R A T}(\bar{\phi})}\right)$ holds.

By the definition of $T_{\bar{\phi}}$ this means that $G_{F \cap \mathbf{R A T}(\bar{\phi})} \subseteq T_{\bar{\phi}}\left(G_{\mathbf{R A T}(\bar{\phi})}\right)$, i.e. that $G_{F \cap \mathbf{R A T}(\bar{\phi})}$ is a post-fixpoint of $T_{\bar{\phi}}$. Hence by Tarski's Fixpoint Theorem $G_{F \cap \mathbf{R A T}(\bar{\phi})} \subseteq T_{\bar{\phi}}^{\infty}$.

But $s_{i}=s_{i}(\omega)$ and $\omega \in F \cap \mathbf{R A T}(\bar{\phi})$, so we conclude by the above inclusion that $s_{i}$ is an element of the $i$ th component of $T_{\bar{\phi}}^{\infty}$. This proves $G_{C B(\bar{\phi})} \subseteq T_{\bar{\phi}}^{\infty}$.

(iii) $T_{\bar{\phi}}^{\infty} \subseteq G_{C K(\bar{\phi})}$.

Recall that $H=\left(T_{1}, \ldots, T_{n}, p_{1}, \ldots, p_{n}\right)$. We first define

- the functions $s_{1}: \Omega \rightarrow T_{1}, \ldots, s_{n}: \Omega \rightarrow T_{n}$,

- an event $E$, 
- the knowledge correspondences $P_{1}, \ldots, P_{n}$.

Suppose $T_{\bar{\phi}}^{\infty}=\left(S_{1}, \ldots, S_{n}\right)$. Choose $j \in[1 . . n]$ such that the set $S_{j_{0}}$ has the largest cardinality among the sets $S_{1}, \ldots, S_{n}$. Define the function $s_{j_{0}}: \Omega \rightarrow T_{j_{0}}$ arbitrarily, but so that it is onto (note that this is possible since by assumption $\left.|\Omega| \geq\left|T_{j_{0}}\right|\right)$ and let $E:=s_{j_{0}}^{-1}\left(S_{j_{0}}\right)$.

Our aim is to ensure that

$$
G_{E}=T_{\bar{\phi}}^{\infty}
$$

So we define each function $s_{k}: \Omega \rightarrow T_{k}$, where $k \neq j_{0}$, in such a way that $s_{k}^{-1}\left(S_{k}\right)=E$. Note that this is possible since $|E| \geq\left|S_{j_{0}}\right| \geq\left|S_{k}\right|$.

Next, we define each knowledge correspondence $P_{i}$ arbitrarily but so that for all $\omega \in E$ we have $P_{i}(\omega)=E$. Then for all $i \in[1 . . n]$

$$
G_{P_{i}(\omega)}=G_{E}
$$

We now show that for all $\omega \in E$ each player $i$ is $\phi_{i}$-rational in $\omega$. So take an arbitrary $\omega \in E$ and $i \in[1 . . n]$. By the definition of the function $s_{i}(\cdot)$ a strategy $s_{i} \in S_{i}$ exists such that $s_{i}=s_{i}(\omega)$. Now, $T_{\bar{\phi}}^{\infty}$ is a fixpoint of $T_{\bar{\phi}}$, so $\phi_{i}\left(s_{i}, T_{\bar{\phi}}^{\infty}\right)$ holds. But $T_{\bar{\phi}}^{\infty}=G_{E}=G_{P_{i}(\omega)}$, so $\phi_{i}\left(s_{i}(\omega), G_{P_{i}(\omega)}\right)$ holds, i.e. player $i$ is indeed $\phi_{i}$-rational in $\omega$.

To complete the proof take now an arbitrary strategy $s_{i} \in S_{i}$. By the definition of the function $s_{i}(\cdot)$ a state $\omega \in E$ exists such that $s_{i}=s_{i}(\omega)$. Further, we just showed that each player $j$ is $\phi_{j}$-rational in $\omega$. But by the definition of the knowledge correspondences $E$ is an evident event, so it is common knowledge in $\omega$ that each player $j$ is $\phi_{j}$-rational in $\omega$. Hence $\omega \in C K(\bar{\phi})$ and consequently $s_{i}$ is an element of the $i$ th component of $G_{C K(\bar{\phi})}$.

This proves that $T_{\bar{\phi}}^{\infty} \subseteq G_{C K(\bar{\phi})}$.

This theorem shows that when each property $\phi_{i}$ is monotonic, the strategy profiles that the players choose in the states in which it is common knowledge that each player $i$ is $\phi_{i}$-rational (or in which each player $i$ is $\phi_{i}$-rational and it is common belief that each player $i$ is $\phi_{i}$-rational), are exactly those that remain after the iterated elimination of the strategies that are not $\phi_{i}$-optimal. It generalizes corresponding results established for finite strategic games (for their survey see Battigalli and Bonanno [1999]) to the case of arbitrary strategic games and arbitrary monotonic properties $\phi_{i}$.

In Chen, Long and Luo [2005], Lipman [1994] and Apt [2007] examples are provided showing that for the properties of strict dominance (namely $s d^{g}$ ) and best response (namely $b r^{g}$ ) in general transfinite iterations (i.e., iterations beyond $\omega_{0}$ ) of the corresponding operator are necessary to reach the outcome. So to achieve equalities in the above theorem transfinite iterations of the $T_{\bar{\phi}}$ operator are necessary.

By instantiating $\phi_{i}$ s to specific properties we get instances of the above result that relate to specific definitions of rationality. Before we do this we establish another result that will apply to another class of properties $\phi_{i}$.

Consider the following natural property of the underlying functions $s_{i}(\cdot) \mathrm{s}$ :

A For each strategy $s_{i}$ from $H$ a state $\omega \in \Omega$ exists such that $s_{i}=s_{i}(\omega)$.

Theorem 2 Suppose that property $\boldsymbol{A}$ holds and

$$
\phi_{i}\left(s_{i},\left(\left\{s_{1}\right\}, \ldots,\left\{s_{n}\right\}\right)\right) \text { for all } i \in[1 . . n] \text { and } s_{i} \in T_{i} \text {. }
$$

Then

$$
G_{C K(\bar{\phi})}=G_{C B(\bar{\phi})}=H \text {. }
$$

Proof. As noted in the proof of Theorem 1, for all $\bar{\phi}$ we have $G_{C K(\bar{\phi})} \subseteq G_{C B(\bar{\phi})}$. So it suffices to prove that $H \subseteq G_{C K(\phi)}$.

So take a strategy $s_{i}$ of player $i$ in $H$. By property A a state $\omega$ exists such that $s_{i}=s_{i}(\omega)$. Choose for each player $j$ a knowledge correspondence $P_{j}$ such that $P_{j}(\omega)=\{\omega\}$. Then

$$
G_{P_{j}(\omega)}=\left(\left\{s_{1}(\omega)\right\}, \ldots,\left\{s_{n}(\omega)\right\}\right)
$$

and, on the account of (3), each player $j$ is $\phi_{j}$-rational in $\omega$.

By the choice of the knowledge correspondences $\{\omega\}$ is an evident event. Hence it is common knowledge in $\omega$ that each player $j$ is $\phi_{j}$-rational in $\omega$. So by definition $s_{i}$ is an element of the $i$ th component of $C K_{\phi}$.

Note that any property $\phi_{i}$ that satisfies (3) and is not trivial (that is, for some strategy $s_{i}, \phi_{i}\left(s_{i}, H\right)$ does not hold) is not monotonic.

\section{$5 \quad \ldots$ and their consequences}

Let us analyze now the consequences of the above two theorems. Consider first Theorem 1. The following lemma, in which we refer to the properties introduced in Section 3, clarifies the matters.

Lemma 2 The properties $s d^{g}$, $\mathrm{msd}^{g}$ and $b r^{g}$ are monotonic. 
Proof. Straightforward.

So Theorem 1 applies to the above three properties. (Note that $b r^{g}$ actually comes in three 'flavours' depending on the choice of beliefs.) Strict dominance in the sense of $s d^{g}$ is studied in Chen, Long and Luo [2005], while $b r^{g}$ corresponds to the rationalizability notion of Bernheim [1984].

To see the consequences of Theorem 2 note the following lemma.

Lemma 3 The properties $s d^{l}$, msd $^{l}$ and $b r^{l}$ satisfy (3).

Proof. Straightforward.

So Theorem 2 shows that the 'customary' concepts of strict dominance, $s d^{l}$ and $m s d^{l}$ and the 'local' version of the best response property $b r^{l}$ cannot be justified in the used epistemic framework as 'stand alone' concepts of rationality. Indeed, this theorem shows that common knowledge that each player is rational in one of these three senses does not exclude any strategy.

What can be done is to justify these concepts as consequences of the common knowledge of rationality defined in terms of $b r^{g}$, the 'global' version of the best response property, Namely, we have the following result. When each property $\phi_{i}$ equals $\phi$, we write here $C K(\phi)$ instead of $C K(\bar{\phi})$ and analogously for $C B$.

Theorem 3 For all games $H$

$$
G_{C K\left(b r^{g}\right)}=G_{C B\left(b r^{g}\right)} \subseteq T_{s d^{l}}^{\infty},
$$

where we take as the set of beliefs the set of joint strategies of the opponents.

Proof. By Lemma 2 and Theorem $1 G_{C K\left(b r^{g}\right)}=$ $G_{C B\left(b r^{g}\right)}=T_{b r^{g}}^{\infty}$. Each best response to a joint strategy of the opponents is not strictly dominated, so for all restrictions $G$

$$
T_{b r^{g}}(G) \subseteq T_{s d^{g}}(G)
$$

and also

$$
T_{s d^{g}}(G) \subseteq T_{s^{l}}(G) .
$$

So by Lemma $1 T_{b r}^{\infty} \subseteq T_{s d^{l}}^{\infty}$, which concludes the proof.

The above result formalizes and justifies in the epistemic framework used here the often used statement:

common knowledge of rationality implies that the players will choose only strategies that survive the iterated elimination of strictly dominated strategies for games with arbitrary strategy sets and transfinite iterations of the elimination process.

In the case of finite games we have the following known result. For a proof using Harsanyi type spaces see Brandenburger and Friedenberg [2006].

Theorem 4 For all finite games $H$

$G_{C K\left(b r^{g}\right)}=G_{C B\left(b r^{g}\right)} \subseteq T_{m s d^{l}}^{\infty}$,

where we take as the set of beliefs the set of joint mixed strategies of the opponents.

Proof. The argument is analogous as in the previous proof but relies on a subsidiary result.

Again by Lemma 2 and Theorem $1 G_{C K\left(b r^{g}\right)}=$ $G_{C B\left(b r^{g}\right)}=T_{b r^{g}}^{\infty}$. Further, for all restrictions $G$

$$
T_{b r^{g}}(G) \subseteq T_{b r^{l}}(G)
$$

and

$$
T_{b r^{l}}(G) \subseteq T_{b r c^{l}}(G),
$$

where $b r c^{l}$ stands for the best response property w.r.t. the correlated strategies of the opponents. So by Lemma $1 T_{b r^{g}}^{\infty} \subseteq T_{b r c l}^{\infty}$.

But by the result of Osborne and Rubinstein [1994, page 60] (that is a modification of the original result of Pearce [1984]) for all restrictions $G$ we have $T_{b r c^{l}}(G)=T_{m s d^{l}}(G)$, so $T_{b r c^{l}}^{\infty}=T_{m s d^{l}}^{\infty}$, which yields the conclusion.

\section{Acknowledgements}

We acknowledge helpful discussions with Adam Brandenburger, who suggested Theorems 3 and 4, and with Giacomo Bonanno who, together with a referee of Apt [2007] suggested to incorporate common beliefs in the analysis. Joe Halpern pointed us to Monderer and Samet [1989]. Jonathan Zvesper provided helpful comments on the paper.

\section{References}

K. R. Apt

[2007] The many faces of rationalizability, Topics in Theoretical Economics, 7(1). Article 1, 39 pages. Available from http://arxiv.org/abs/ cs.GT/0608011.

\section{R. Aumann}

[1976] Agreeing to disagree, The Annals of Statistics, 4, pp. $1236-1239$

[1987] Correlated equilibrium as an expression of Bayesian rationality, Econometrica, 55, pp. 118. 


\section{P. Battigalli and G. Bonanno}

[1999] Recent results on belief, knowledge and the epistemic foundations of game theory, Research in Economics, 53, pp. 149-225.

\section{J. VAN BENTHEM}

[2007] Rational dynamics and epistemic logic in games, International Journal of Game Theory. To appear.

B. D. BERNHEIM

[1984] Rationalizable strategic behavior, Econometrica, 52, pp. 1007-1028.

P. Blackburn, M. De Rijke, and Y. Venema

[2001] Modal Logic, Cambridge University Press.

A. Brandenburger and E. Dekel

[1987] Rationalizability and correlated equilibria, Econometrica, 55, pp. 1391-1402.

\section{A. Brandenburger and A. Friedenberg}

[2006] Intrinsic correlation in games. Working paper. Available from http://pages.stern.nyu.edu/ abranden.

A. Brandenburger, A. Friedenberg, and H. Keisler

[2004] Admissibility in games. Working paper. Revised October 2006. Available from http://pages. stern.nyu.edu/ abranden.

Y.-C. Chen, N. V. Long, And X. Luo

[2005] Iterated strict dominance in general games. Available from http://www.sinica.edu.tw/ xluo/pa10.pdf.

\section{Dufwenberg and M. Stegeman}

[2002] Existence and uniqueness of maximal reductions under iterated strict dominance, Econometrica, 70, pp. 2007-2023.

J. ELy AND M. PESKI

[2006] Hierarchies of belief and interim rationalizability, Theoretical Economics, 1, pp. 1965. Available from http://ideas.repec.org/ a/the/publsh/163.html.

\section{A. Heifetz And D. SAmet}

[1998] Knowledge spaces with arbitrarily high rank, Games and Economic Behavior, 22, pp. 260273

B. L. Lipman

[1991] How to decide how to decide how to ... Modeling limited rationality, Econometrica, 59, pp. $1105-1125$.

[1994] A note on the implications of common knowledge of rationality, Games and Economic Behavior, 6 , pp. $114-129$.

R. D. LuCE AND H. RAIfFA

[1957] Games and Decisions, John Wiley and Sons, New York.

\section{P. Milgrom and J. Roberts}

[1990] Rationalizability, learning, and equilibrium in games with strategic complementarities, Econometrica, 58, pp. $1255-1278$.

D. Monderer And D. SAmet

[1989] Approximating common knowledge with common beliefs, Games and Economic Behaviour 1, pp. 170-190.

M. J. Osborne And A. Rubinstein

[1994] A Course in Game Theory, The MIT Press, Cambridge, Massachusetts.

\section{G. PeArCe}

[1984] Rationalizable strategic behavior and the problem of perfection, Econometrica, 52, pp. 1029 1050 .

\section{T.-C. TAN AND S. Werlang}

[1988] The Bayesian foundations of solution concepts of games, Journal Of Economic Theory, 45, pp. $370-391$.

\section{A. TARSKI}

[1955] A lattice-theoretic fixpoint theorem and its applications, Pacific J. Math, 5, pp. 285-309. 